\title{
Modelling and Simulation of Three Level Inverters for Main Drive of the Plate Mill Rolling Stand
}

\author{
Vadim R. Gasiyarov ${ }^{1}$, Andrey A. Radionov ${ }^{1}$ and Alexander S. Maklakov ${ }^{1}$ \\ ${ }^{1}$ South Ural State University, 454080, 76 Lenin Avenue, Chelyabinsk, Russia
}

\begin{abstract}
This paper addresses a modelling and a simulation of three level inverters for main drive of the plate mill rolling stand. The main purpose of the article is to develop of a mathematical description of the three level voltage source inverter based on a space vector pulse width modulation and to simulate a consumption current by the main electric drive based on 18-pulse rectification circuit. A twelve-megawatt synchronous motor as a load has been used. The modelling results were simulated at the Matlab/Simulink program. It can allow us to estimate an electromagnetic compatibility of the main electric drive based on transients of the consumption current. The research results can be used to assess the electromagnetic compatibility of powerful three level natural point clamped converters with different types of the space vector pulse width modulation on a load or a grid.
\end{abstract}

\section{Introduction}

Nowadays medium voltage reversible electric drives based on synchronous motors and power converters, which consist of active front end rectifiers (AFEs) and voltage source inverters (VSIs), are widely used in electrotechnical systems of rolling stands. Such consumers are able to create a bidirectional power flow at a low negative effect on the grid. Usually medium voltage drives have a high rated power, so a modelling and a researching of these drives are more effective to increase an energy-efficiency of real industrial objects. Power quality and the energy efficiency are very important factors to keep the production costs [1].

Very often the medium voltage AFEs and VSIs have a neutral point clamped (NPC) stricture. A parallel connecting of the AFEs or the VSIs is commonly used to create more than six-pulse rectifier circuits. For example, several transformers with a shift angle of secondary voltages on $20^{\circ}, 0^{\circ}$ and $-20^{\circ}$ for 18 -pulse circuit and $15^{\circ}$, $0^{\circ},-15^{\circ}$ and $30^{\circ}$ for 24-pulse circuit can be applied [2].

A structural feature of powerful AFEs and VSIs is three level NPC topology. It allows one to improve an electromagnetic capability with the grid or a load at a low swathing frequency of semiconductors modules. Total harmonic distortional (THD) of output current or voltage for three level NPC converters is a small magnitude due to the increasing of output voltage levels. High voltage insulate gate bipolar transistors (HV IGBTs), integrated gate-commutated thyristors (IGCTs) and injection enhanced gate transistors (IEGTs) are generally used as the semiconductors modules for AFEs and VSIs [4].

A control of the modules is done by the pulse width modulation (PWM). The most common PWM technology is a space vector (SVPWM) and a selective harmonics elimination (SHE PWM). In AFEs operate based on the SHEPWM and in VSIs are used the SVPWM $[5,6]$.

\section{Problem definition}

The main purpose of this paper is to develop a mathematical model of a power circuit of the electric drive of the plate mill rolling stand based on three level NPC inverters with SVPWM. Additional purpose is to evaluate the electromagnetic compatibility of consumption current by the synchronous motor.

\section{Development of a mathematical description}

\subsection{Typical features of the main drive of the plate mill rolling stand}

Generally, a main electric drive of plate mill rolling stands is system, in which synchronous motor of each mill roll are connected to the grid through several medium voltage three level NPC converters. The NPC converters consist of AFEs and VSIs. The maximal power of each converter can achieve more than 20 MVA. 18-pulse or 24-pulse rectification circuits are typically used to improve the quality of direct voltage and to reduce the harmonic level of the consumption current. The inductor with high voltage short circuit are applied at the input of motors [7-11]. The characteristic power circuit for main electric drive of plate mill rolling stand is shown in Figure 1. 


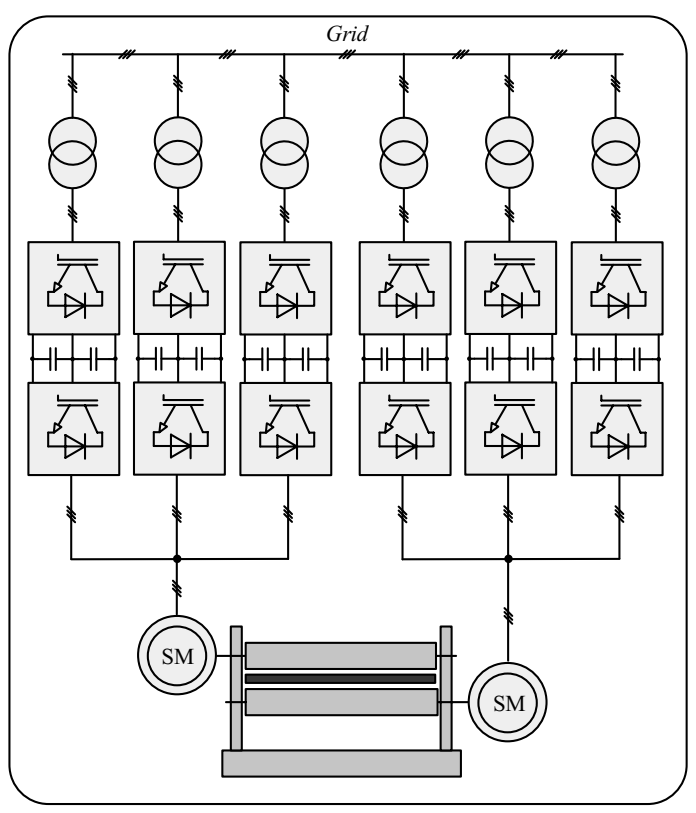

Figure 1. Characteristic power circuit for main electric drive of plate mill rolling stand

\subsection{Modelling of a three-level inverter}

There are a lot of control methods of three-level inverters, but at present the SVPWM technology is widely used. The exponential increase of digital signal processors (DSPs) has allowed one to develop the complex algorithms SVPWM for multilevel NPC inverters. It is possible to reduce switching losses using low switching frequency of semiconductor modules [12-14]. The threelevel VSI based on the NPC structure is shown in Figure 2.

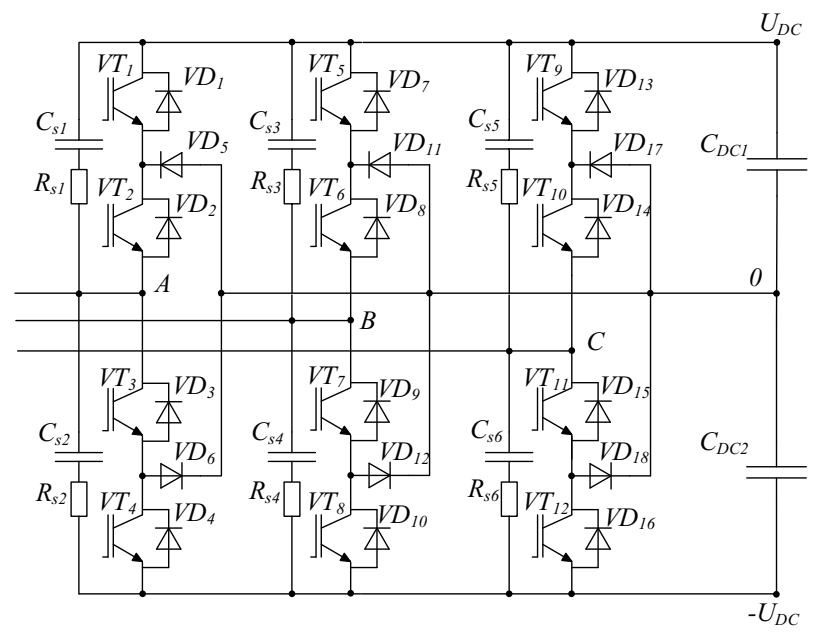

Figure 2. Three level VSI based on NPC structure

The three level VSI in Figure 1 contains twelve HVIGBT or IEGT transistors $\left(V T_{1} \ldots V T_{12}\right)$ and eighteen diodes $\left(V D_{1} \ldots V D_{18}\right)$. Two equivalent capacitors $C_{D C l}$ and $C_{D C 2}$ create a neutral point 0 . Snubbers consist of the resistors $\left(R_{S I} \ldots R_{S 6}\right)$ and the capacitors $\left(C_{S 1} \ldots C_{S 6}\right)$, which are parallel connected the semiconductor modules [15].

An output phase voltage of a VSI has magnitudes $+U_{d d} / 2,0$, and $-U_{d d} / 2$. If the symmetric phase system is considered, all curves of the phase voltages have an odd harmonic spectrum.

According to Kirchhoff's laws, the system equations have been given and written based on snubbers current $i_{S I}$ and $i_{S 2}$, and current $i_{1}, i_{2}, i_{0}$ flowing through the semiconductor modules for all operating conditions:

$$
\left\{\begin{aligned}
i_{S 1}= & \frac{u_{S 2}-u_{S 1}+u_{D C 1}+u_{D C 2}+R_{S} \cdot i_{A}}{2 \cdot R_{S}}- \\
& -\frac{u_{S 1}+u_{S 1}+u_{D C 1}+u_{D C 2}+R_{S} \cdot i_{A}}{2 \cdot R_{S}+4 \cdot R_{o n}} \\
i_{S 2} & =\frac{u_{S 1}-u_{S 2}+u_{D C 1}+u_{D C 2}+2 \cdot R_{o n} \cdot i_{A}}{2 \cdot R_{S}+4 \cdot R_{o n}}- \\
& -\frac{u_{S 1}+u_{S 1}-u_{D C 1}-u_{D C 2}}{2 \cdot R_{S}} \\
i_{1} & =\frac{u_{S 1}+u_{S 2}-u_{D C 1}-u_{D C 2}+R_{S} \cdot i_{A}}{R_{S}+2 \cdot R_{o n}}
\end{aligned}\right.
$$

$$
\begin{aligned}
& \int i_{S 1}=\frac{u_{S 2}-u_{S 1}+u_{D C 1}+u_{D C 2}+2 \cdot R_{o n} \cdot i_{A}}{2 \cdot R_{S}-4 \cdot R_{o n}}- \\
& -\frac{u_{S 1}+u_{S 1}-u_{D C 1}-u_{D C 2}}{2 \cdot R_{S}} \\
& i_{S 2}=\frac{R_{o n}\left(u_{S 1}+u_{S 2}-u_{D C 1}-u_{D C 2}\right)-R_{S}\left(u_{S 2}+R_{o n} \cdot i_{A}\right)}{R_{S}{ }^{2}+2 \cdot R_{S} \cdot R_{o n}} ; \\
& i_{2}=\frac{u_{S 2}-u_{S 1}+u_{D C 1}+u_{D C 2}+R_{S} \cdot i_{A}}{R_{S}-2 \cdot R_{o n}} \\
& i_{S 1}=\frac{u_{S 2}+u_{D C 1}-u_{S 1}-u_{D C 2}-2 \cdot R_{o n} \cdot i_{A}}{2 \cdot R_{S}+4 \cdot R_{o n}}- \\
& -\frac{u_{S 1}+u_{S 1}-u_{D C 1}-u_{D C 2}}{2 \cdot R_{S}} \\
& \left\{i_{S 2}=\frac{R_{o n} \cdot\left(u_{D C 1}-u_{D C 2}-u_{S 1}-u_{S 2}\right)}{R_{S}{ }^{2}+2 \cdot R_{S} \cdot R_{o n}} \quad ;\right. \\
& +\frac{R_{S} \cdot\left(u_{D C 2}-u_{S 2}+R_{o n} \cdot i_{A}\right)}{R_{S}{ }^{2}+2 \cdot R_{S} \cdot R_{o n}} \\
& i_{0}=\frac{u_{S 2}-u_{S 1}+u_{D C 1}-u_{D C 2}+R_{S} \cdot i_{A}}{R_{S}+2 \cdot R_{\text {on }}} \\
& \left\{\begin{array}{l}
i_{S 1}=-\frac{1}{2} \cdot\left(i_{A}+\frac{u_{S 1}+u_{S 2}-u_{D C 1}-u_{D C 2}}{2 \cdot R_{S}}\right) \\
i_{S 2}=\frac{1}{2} \cdot\left(i_{A}-\frac{u_{S 1}+u_{S 2}-u_{D C 1}-u_{D C 2}}{2 \cdot R_{S}}\right)
\end{array},\right.
\end{aligned}
$$

where $R_{S}=R_{S 1}=R_{S 2}-$ active resistance of the first and the second snubbers; $R_{o n}=R_{o n V T 1}+R_{o n V T 2}=R_{o n V D 1}+$ $R_{\text {onVD2 }}$ - total active resistance of the first and the second transistors or diodes; $i_{S 1}$ and $i_{S 2}$ - snubber currents; $i_{1}-$ current flowing through the first and the second transistors or diodes; $u_{D C 1}, u_{D C 2}-$ direct current (DC) voltages of the equivalent capacitors $C_{D C l}$ and $C_{D C 2} ; u_{S I}$ and $u_{S 2}$ - snubber voltages; $i$ - load current of the phase A. 


\subsection{Modelling of SVPWM}

The output three-phase voltages of the VSI has the following system equation:

$$
\left\{\begin{array}{l}
U_{A 0}=\sum_{n=1,3, \ldots, \infty}^{\infty} U_{n} \cdot \sin (n \cdot \omega \cdot t) \\
U_{B 0}=\sum_{n=1,3, \ldots, \infty}^{\infty} U_{n} \cdot \sin \left(n \cdot \omega \cdot t-\frac{2 \cdot \pi}{3}\right) \\
U_{C 0}=\sum_{n=1,3, \ldots, \infty}^{\infty} U_{n} \cdot \sin \left(n \cdot \omega \cdot t+\frac{2 \cdot \pi}{3}\right) \\
U_{n}=m \cdot \frac{2 \cdot U_{d c}}{\pi \cdot n} \cdot\left[\sum_{i=1}^{n}(-1)^{i+1} \cdot \cos \left(n \cdot \alpha_{i}\right)\right]
\end{array}\right.
$$

where $\alpha_{i}$ - angle switching $\left(0<\alpha_{i}<180^{\circ}\right)$; $n-$ harmonics number $(1,3, \ldots, \infty) ; \omega$ - angular frequency of synchronous motor; $m$ - modulation index; $U_{A 0}, U_{B 0}, U_{C 0}$ - output phase voltage of the VSI; $t$ - time.

If first harmonic of the system (1) is considered, the space vector theory can be used to write the following system equation [16]:

$$
\left\{\begin{array}{l}
U_{A 0}=U_{1} \cdot \sin (\omega \cdot t) \\
U_{B 0}=U_{1} \cdot \sin \left(\omega \cdot t-\frac{2 \cdot \pi}{3}\right) \\
U_{C 0}=U_{1} \cdot \sin \left(\omega \cdot t+\frac{2 \cdot \pi}{3}\right)
\end{array}\right.
$$

Based on the system equation (2) we can use the space vector theory and apply the following expression:

$$
\bar{U}=\frac{2}{3} \cdot\left(U_{A 0} \cdot e^{j \cdot 0}+U_{B 0} \cdot e^{j \cdot \frac{2 \cdot \pi}{3}}+U_{C 0} \cdot e^{j \cdot \frac{4 \cdot \pi}{3}}\right)
$$

The equation (3) allows us to determine a reference space vector for an each switching state of the three-level VSI [17]. In Figure 3, the space vector diagram of three level NPC inverters is presented.

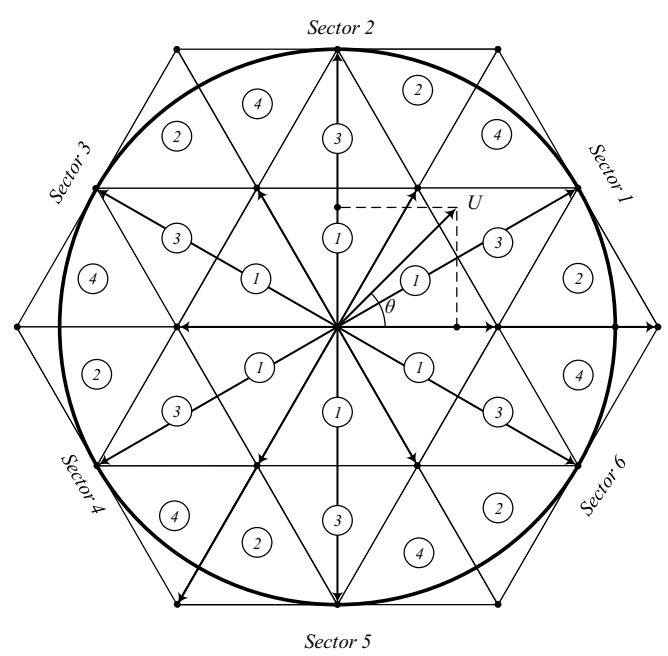

Figure 3. Space vector diagram of three level NPC inverters
The space vector diagram in Figure 3 is a symmetric hexagon with shift on $60^{\circ}$ for the each sectors. The angle $\theta$ determines the sector number based on the following conditions:

$$
\begin{gathered}
0^{\circ} \leq \theta<60^{\circ}-\text { Sector } 1 ; \\
60^{\circ} \leq \theta<120^{\circ}-\text { Sector } 2 ; \\
120^{\circ} \leq \theta<180^{\circ}-\text { Sector } 3 ; \\
180^{\circ} \leq \theta<240^{\circ}-\text { Sector } 4 ; \\
240^{\circ} \leq \theta<300^{\circ}-\text { Sector } 5 ; \\
300^{\circ} \leq \theta<360^{\circ}-\text { Sector } 6 .
\end{gathered}
$$

The times calculation $T_{1}, T_{2}, T_{3}$ for each sector and region are as the following equations $[18,19]$ :

for region 1:

$$
\begin{aligned}
& T_{1}=T_{S}-T_{2}-T_{3}=T_{S} \cdot\left(1-3 \frac{U}{U_{d c}} \cos (\theta)-\sqrt{3} \frac{U}{U_{d c}} \sin (\theta)\right) \\
& T_{2}=T_{S} \cdot\left(3 \frac{U}{U_{d c}} \cos (\theta)-\sqrt{3} \frac{U}{U_{d c}} \sin (\theta)\right) \\
& T_{3}=T_{S} \cdot 2 \sqrt{3} \frac{U}{U_{d c}} \sin (\theta)
\end{aligned}
$$

for region 2:

$$
\begin{aligned}
& T_{1}=T_{S}-T_{2}-T_{3}=T_{S} \cdot\left(2-3 \frac{U}{U_{d c}} \cos (\theta)-\sqrt{3} \frac{U}{U_{d c}} \sin (\theta)\right) \\
& T_{2}=T_{S} \cdot\left(3 \frac{U}{U_{d c}} \cos (\theta)-\sqrt{3} \frac{U}{U_{d c}} \sin (\theta)-1\right) \\
& T_{3}=T_{S} \cdot 2 \sqrt{3} \frac{U}{U_{d c}} \sin (\theta)
\end{aligned}
$$

for region 3:

$$
\begin{aligned}
& T_{1}=T_{S}-T_{2}-T_{3}=T_{S} \cdot\left(3 \frac{U}{U_{d c}} \cos (\theta)+\sqrt{3} \frac{U}{U_{d c}} \sin (\theta)-1\right) \\
& T_{2}=T_{S} \cdot\left(1-3 \frac{U}{U_{d c}} \cos (\theta)+\sqrt{3} \frac{U}{U_{d c}} \sin (\theta)\right) \\
& T_{3}=T_{S} \cdot\left(1-2 \sqrt{3} \frac{U}{U_{d c}} \sin (\theta)\right)
\end{aligned}
$$

for region 4:

$$
\begin{aligned}
& T_{1}=T_{S}-T_{2}-T_{3}=T_{S} \cdot\left(2-3 \frac{U}{U_{d c}} \cos (\theta)-\sqrt{3} \frac{U}{U_{d c}} \sin (\theta)\right) \\
& T_{2}=T_{S} \cdot\left(3 \frac{U}{U_{d c}} \cos (\theta)-\sqrt{3} \frac{U}{U_{d c}} \sin (\theta)\right) \\
& T_{3}=T_{S} \cdot\left(2 \sqrt{3} \frac{U}{U_{d c}} \sin (\theta)-1\right)
\end{aligned}
$$

The example of space vector formation in the Sector 1 and the region 1 is shown in Figure 4. 


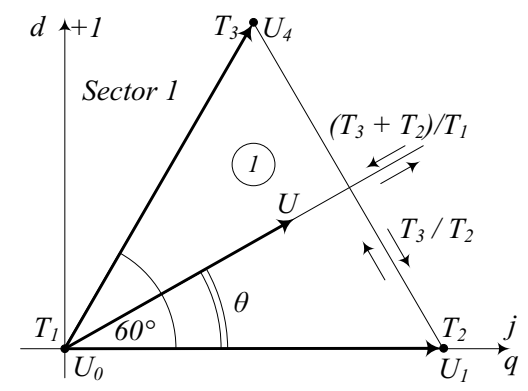

Figure 4. Space vector formation

A ratio of the times $T_{1}, T_{2}$, and $T_{3}$ regulate the space vector depth and the angle by using the following expression:

$$
\begin{gathered}
\frac{T_{3}+T_{2}}{T_{1}}-\text { the space vector depth; } \\
\frac{T_{3}}{T_{2}}-\text { the space vector angle. }
\end{gathered}
$$

\section{Simulation of the main drive of the plate mill rolling stand}

The simulation model of the main drive of the plate mill rolling stand based on the three level NPC inverters with SVPWM in Matlab/Simulink was developed. The block diagram of this model is shown in Figure 5.



Figure 5. Simulation model of the main drive of the plate mill rolling
A closed loop system of three level NPC inverter has been developed on the basis of a classical structure orientated according to the direction of angular position of synchronous motor, which in the articles [18, 20, 21] has been considered. Synthesis of regulators was developed using the step-by-step correction principle. The modeling results of a consumption phase current by the synchronous motor and the output phase-to-phase voltage of VSI are shown in Figure 6 and Figure 7.

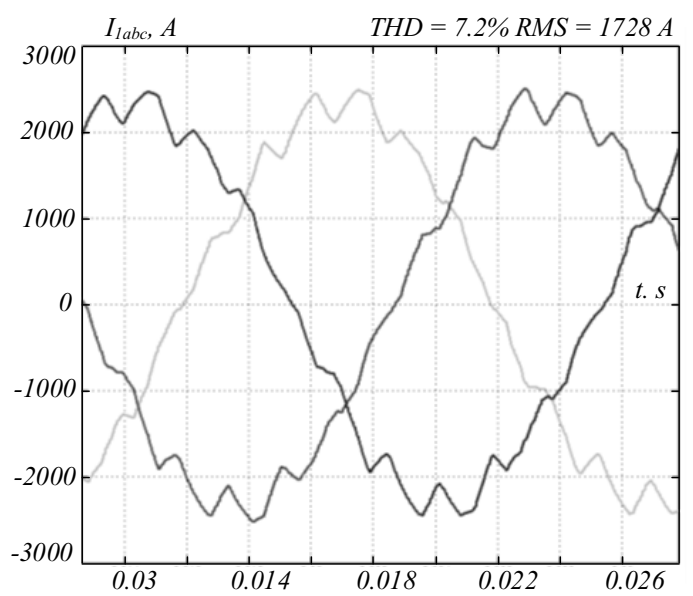

Figure 6. Consumption phase current by the synchronous motor

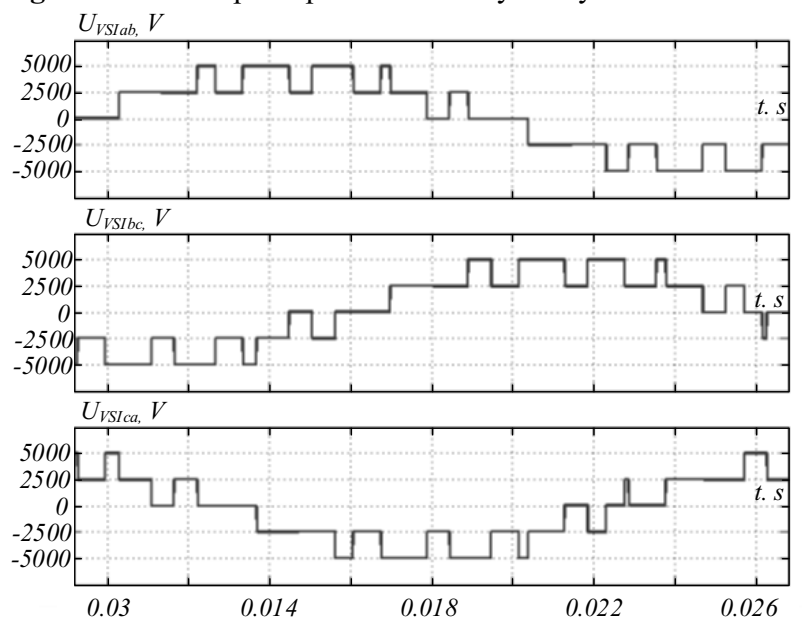

Figure 7. Output phase-to-phase voltage of VSI

\section{Conclusion}

The mathematical description and the simulation of three level VSI based on NPC structure for main drive of the plate mill rolling stand have been made in the article. The mathematical description of the three level VSI based on the SVPWM and to simulate the consumption current by the main electric drive based on 18-pulse rectification circuit have been developed. The modelling results were simulated at the Matlab/Simulink program. This model can be used to estimate the electromagnetic compatibility of the main electric drive based on transients of the consumption current. The research results can be employed to assess the electromagnetic compatibility of the powerful three level NPC converters with different types of the SVPWM on the load or the grid. 


\section{References}

1. Leopoldo G. Franquelo, Jose Rodríguez, Jose I. Leon, Samir Kouro, Ramon Portillo and Maria A.M. Prats, IEEE industrial electronics magazine, 2, 28-39 (2008)

2. J. Rodriguez, J.-S. Lai, F.Z. Peng, IEEE Trans. Ind. Electron., 49, 4, 724-738 (2002)

3. J. Rodriguez, S. Bernet, B. Wu, J. Pontt, and S. Kouro, IEEE Trans. Ind. Electron., 54, 6, 2930-2945 (2007)

4. A. Nabae, I. Takahashi, and H. Akagi, IEEE Trans. Ind. Applicat., 1A-17, 5, 518-523 (1981)

5. ABD Almula G. M. Gebreel, Simulation and implementation of two-level and three-level inverters by matlab and RT-LAB, 136 (2011)

6. Mr. Anupam Mishra, Mr. Sunil Panda, Mr. B. Srinivas, Control of voltage source inverters using $P W M / S V P W M$ for adjustable speed drive applications, 43 (2009)

7. A.S. Maklakov, A.A. Radionov, Actual Problems of Electronics Instrument Engineering (APEIE), 2014 12th International Conference, 770-774 (2014)

8. A.S. Maklakov, A.A. Radionov, Russian Internet Journal of Industrial Engineering, 2, 40-47 (2013)

9. B. Wu, Y. Lang, N. Zargari, S. Kouro, Power Conversion and Control of Wind Energy Systems, $453(2011)$

10. B. K. Bose, Power Electronics and Motor Drives: Advances and Trends, (2006)

11. B. Wu, High-Power Converters and AC Drives, (2006)
12. Dong-Myung Lee, Jin-Woo Jung, and Sang-Shin Kwak, Journal of Power Electronics, 11, 5, 688-696 (2011)

13. Haibing $\mathrm{Hu}$, Wenxi Yao, and Zhengyu Lu, Trans. on Power Electr., 22, 6, 2234-2244 (2007)

14. Remus Teodorescu, Marco Liserre, Pedro Rodriguez. Grid converters for photovoltaic and wind power systems, 398 (2011)

15. T.R. Khramshin, D.S. Krubtsov, G.P. Kornilov, Russian Internet Journal of Electrical Engineering, 1, 1, 3-7 (2014)

16. L. Malesani, L. Rossetto, P. Tomasin, P. Tenti, IEEE Trans. on Ind. App., 31, 2, 287-292 (1995)

17. V. Blasko, V. Kaura. IEEE Trans. Ind. Electron., 12, 1, 116-123 (1997)

18. A.S. Maklakov, Russian Internet Journal of Industrial Engineering, 3, 16-25 (2014)

19. A.S. Maklakov, A.A. Radionov, Proceedings of the 2015 IEEE North West Russia Section Young Researchers in Electrical and Electronic Engineering Conference, ElConRusNW 2015, 236240 (2015)

20. A.A. Radionov, A.S. Maklakov, E.A. Karyakina, Proceedings 2015 International Siberian Conference on Control and Communications, SIBCON 2015, (2015).

21. A.A. Radionov, A.S. Maklakov, V.R. Gasiyarov, Proceedings of 2014 International Conference on Mechanical Engineering, Automation and Control Systems, MEACS 2014, (2014). 